\title{
ENERGY SPECTRUM IN QUANTUM DOTS OF LEAD AND TIN CHALCOGENIDES SEMICONDUCTING COMPOUNDS
}

\author{
V.K. Dugaev, V.I. Litvinov, P.P. Petrov \\ Chernovtsy Department of the Institute of Materials Science Problems \\ Ukrainian Academy of Sciences \\ I. Wilde 5, 274001 Chernovtsy, Ukraine \\ O.A. Mironov, O.N. NaShCHEKINA \\ Institute of Radiophysics and Electronics, Ukrainian Academy of Sciences \\ Acad. Proskura 12, 310085 Kharkov, Ukraine
}

AND M. OszWądowski

Technical University of Poznań, Piotrowo 3, 60-965 Poznań, Poland

The energy spectrum of a quantum dot made from IV-VI narrow gap semiconductors is studied. The calculations of the energy levels as functions of the dot radius are performed. When the anisotropy of the bare energy spectrum is strong, the energy levels are calculated using Fal'kovskii's adiabatic approximation for multiband systems. When the quantum dot material has an inverted band gap with respect to the host, the low-energy states within the fundamental gap are shown to arise.

PACS numbers: $71.50 .+t, 71.90 .+q$

The present day semiconductor technology is able to produce quantum dots which are effectively the objects of zero dimensionality [1-3]. The size quantization of the energy spectrum in quantum dots takes place in all three dimensions. Here we study the energy spectrum of a quantum dot made of the narrow gap semiconductors and their solid solutions. The bulk crystal of this type has nonparabolic spectrum. The complexity of spectrum makes it nontrivial to solve the size quantization problem. In reality, the characteristic width $L$ of the three-dimensional quantum well (i.e. the dot size) is not too small, $L \gg a$, where $a$ is the lattice constant. Thus, the envelope function approximation can be used. It implies that the basis wave functions contain as a set of multipliers the wave functions at the extremum point for only valence and conduction bands.

We restrict ourselves to investigation of the type-I model of heterojunction, in which the relevant effective potential is $E_{\mathrm{g}}(r)$. The possible variation of other 
parameters including the crystal potential $V(r)$ (or band offsets) is neglected. It is worth mentioning that the band offsets in compounds under consideration are not determined definitively so far.

Thus, we study the eigenvalue and eigenfunction problem with the following Dirac Hamiltonian:

$$
H=\frac{\tau_{z}}{2} E_{\mathrm{g}}(r)-\mathrm{i} v_{t} \tau_{x}\left(\sigma_{x} \partial_{x}+\sigma_{y} \partial_{y}\right)-\mathrm{i} v_{z} \tau_{x} \sigma_{z} \partial_{z},
$$

describing the electrons and holes in the vicinity of the $L$ extremum point of Brillouin zone. Here $\sigma$ and $\boldsymbol{\tau}$ are the Pauli matrices acting in spin and band spaces respectively, $v_{t}$ and $v_{z}$ are the interband interaction parameters.

In real IV-VI crystals the ratio $v_{t} / v_{z}$ varies from about 10 in $\mathrm{PbTe}$ to 1 in $\mathrm{PbS}$ [4], whereas the gap parameter $E_{\mathrm{g}}$ can be positive (e.g. $\mathrm{PbTe}$ ) or negative (SnTe). In accordance with that we studied separately two different models. The first one is the fully isotropic model with $v_{t}=v_{z}$ and isotropic function $E_{\mathrm{g}}(r)$. In the framework of such an approximation we investigated the case of non-inverted junction for which $E_{\mathrm{g}}(r)$ docs not change its sign, and the opposite case of band-inverted junction. The second model relates to the strong anisotropy, $v_{t} / v_{z} \gg 1, E_{\mathrm{g}}(r)$ being still isotropic (notice that in certain important cases of anisotropic $E_{\mathrm{g}}(r)$ the problem reduces to this one after suitable scale transformations).

We take the simplest form of $E_{\mathrm{g}}(r)$, namely $E_{\mathrm{g}}(r)=E_{\mathrm{g} 0}$ at $r<R$, and $E_{\mathrm{g}}(r)=E_{\mathrm{g} 1}$ at $r>R$, where $R$ is the dot radius, and assume $E_{\mathrm{g} 0}<E_{\mathrm{g} 1}$. It may correspond to a sphere of the $\mathrm{Pb}_{1-x} \mathrm{Sn}_{x}$ Te compound with some $x=x_{0}$ inside the host of another compound with $x<x_{0}$.

Using the spherical spinor functions, we can get the eigenvalue equation in the form

$$
\begin{aligned}
& k_{1}\left(\frac{E_{\mathrm{g} 0}}{2}-\varepsilon\right) J_{n+1 / 2}(k R)\left[\frac{n+\kappa}{k_{1} R} K_{n+1 / 2}\left(k_{1} R\right)+K_{n-1 / 2}\left(k_{1} R\right)\right] \\
& \quad=k\left(\frac{E_{\mathrm{g} 1}}{2}-\varepsilon\right) K_{n+1 / 2}\left(k_{1} R\right)\left[\frac{n+\kappa}{k R} J_{n+1 / 2}(k R)-J_{n-1 / 2}(k R)\right] .
\end{aligned}
$$

where $J_{\nu}$ and $K_{\nu}(x)$ are the Bessel functions,

$$
\begin{aligned}
& k=\left(\varepsilon^{2}-E_{\mathrm{g} 0}^{2} / 4\right)^{1 / 2} / v, \quad k_{1}=\left(E_{\mathrm{g} 1}^{2} / 4-\varepsilon^{2}\right)^{1 / 2} / v, \quad n=-1 / 2 \pm(\kappa-1 / 2), \\
& \kappa=-l+1 \text { at } j=l+1 / 2, \quad \kappa=l \text { at } j=l-1 / 2,
\end{aligned}
$$

$j$ and $l$ are the total and orbital momenta. The results of numerical computation are presented in Fig. 1 for the following values of parameters: $E_{\mathrm{g} 0}=0.02 \mathrm{eV}$, $E_{\mathrm{g} 1}=0.1 \mathrm{eV}, v=5 \times 10^{-8} \mathrm{eV} \mathrm{cm}$. In case of $\mathrm{Pb}_{1-x} \mathrm{Sn}_{x} \mathrm{Te}$ alloys, it corresponds to $x \approx 0.3$ and $x \approx 0.2$ respectively [4].

The energy levels are plotted as functions of $R$. The levels exist only at $E_{\mathrm{g} 0}<2|\varepsilon|<E_{\mathrm{g} 1}$. The corresponding wave functions are the linear combinations of wave functions belonging to valence and conduction bands with orbital momenta $l$ and $l+1$. The spin-orbital interaction of Hamiltonian (1) yields the splitting of states with different $l$.

In case of the inverted junction (we take $E_{\mathrm{g} 0}<0, \quad E_{\mathrm{g} 1}>0$ for definiteness) the eigenvalue equation has the same form as Eq. (2) with the substitution 


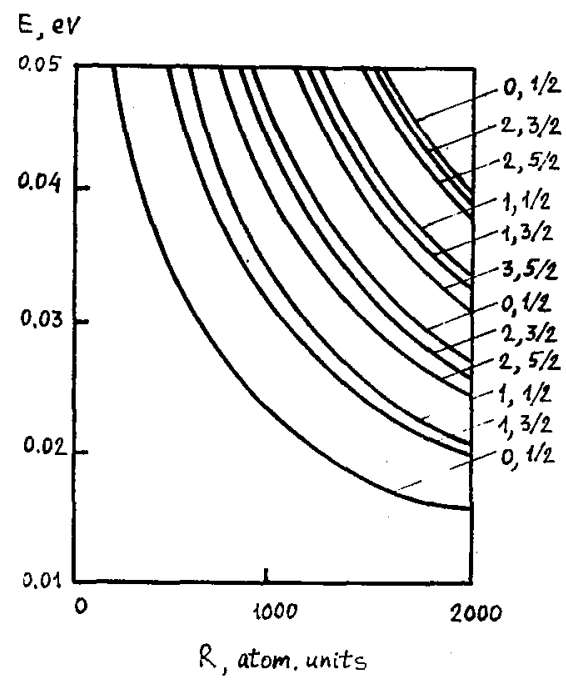

Fig. 1. Dependence of the energies of quantized states versus dot radius. The numbers are the orbital $(l)$ and total $(j)$ momenta.

$J_{\nu} \rightarrow I_{\nu}$, and $k=\left(E_{\mathrm{g} 0}^{2} / 4-\varepsilon^{2}\right)^{1 / 2} / v$. The energy levels can be found by the numerical calculation. The quantization levels lie at $|\varepsilon|<\min \left(\left|E_{\mathrm{g} 0}\right| / 2, E_{\mathrm{g} 1} / 2\right)$, the corresponding wave functions being localized in the vicinity of $r=R$. In the limit $R \rightarrow \infty$ they transform to the known Weyl states with $\varepsilon=0$, predicted earlier for the plane heterojunction [5].

We also performed the calculations of spectrum when anisotropy is strong, $v_{t} / v_{z} \gg 1$. Then the adiabatic approximation can be used in the form developed previously for multiband systems in [6]. The Hamiltonian describing the fast motion is ( $\rho$ is the polar coordinate, $E_{\mathbf{g}}$ is taken positive)

$$
H_{0}=\frac{1}{2} \tau_{z} E_{\mathrm{g}}(\rho)-\mathrm{i} v_{z} \tau_{x}\left(\sigma_{x} \partial_{x}+\sigma_{y} \partial_{y}\right)
$$

It can be diagonalized, leading to the equation

$$
k\left(\frac{E_{\mathrm{g} 0}}{2}+\lambda\right) K_{l}\left(k_{1} R\right) J_{l+1}(k R)=k_{1}\left(\frac{E_{\mathrm{g} 1}}{2}+\lambda\right) K_{l+1}\left(k_{1} R\right) J_{l}(k R),
$$

which determines the effective potential $\lambda(z)$ of slow motion. Here we denoted $R(z)=\left(R_{0}^{2}-z^{2}\right)^{1 / 2}, R_{0}$ is now the dot radius. The second equation of the same form holds for $-\lambda(z)$. There are two wave functions corresponding to these eigenvalues. With them we can get the Hamiltonian for slow motion

$$
H_{1}=\lambda(z) \tau_{z}-\mathrm{i} v_{z} \tau_{x} \partial_{z} .
$$

Since $R_{0} \gg a$, we can approximate the dependence $\lambda(z)$ by $\lambda=\lambda_{0}+\beta z^{2} / 2 R_{0}$, where $\lambda_{0}=\lambda(z=0), \beta \approx \lambda_{0} / R_{0}$. After that, the problem reduces to a one-dimensional Schrödinger equation for a particle of unit mass and energy $E=0$ :

$$
-\frac{1}{2} y^{\prime \prime}+V(z) y=0
$$


where

$$
\begin{aligned}
& V(z)=V_{0}+\frac{1}{2} \omega^{2} z^{2}, \quad V_{0}=\frac{\lambda_{0}^{2}}{2}-\frac{\beta v_{z}^{2}}{2 R_{0}\left(\lambda_{0}+\varepsilon\right)}-\varepsilon^{2}, \\
& \omega^{2}=\left(1+\frac{\beta v_{z}^{2}}{\lambda_{0} R_{0}\left(\lambda_{0}+\varepsilon\right)^{2}}\right) \frac{\lambda_{0} \beta}{R_{0}} .
\end{aligned}
$$

Finally, we obtained the following equation for the energy:

$$
\varepsilon^{2}-\lambda_{0}^{2}+\frac{\beta v_{z}^{2}}{2 R_{0}\left(\lambda_{0}+\varepsilon\right)}=2 n\left[\frac{\lambda_{0} \beta v_{z}^{2}}{R_{0}}\left(1+\frac{\beta v_{z}^{2}}{\lambda_{0} R_{0}\left(\lambda_{0}+\varepsilon\right)^{2}}\right)\right]^{1 / 2},
$$

where $n$ is the quantum number of the longitudinal motion, $n=1 / 2,3 / 2, \ldots$ At $\lambda_{0} R_{0} / v_{z} \gg 1$ we can write approximately $\varepsilon^{2}=\lambda_{0}^{2}+2 n\left(v_{z}^{2} \lambda_{0} \beta / R_{0}\right)^{1 / 2}$.

In conclusion, we obtained the energy spectrum of a spherical quantum dot in different cases. The discrete levels can be observed experimentally only if the number of them is small. As is seen from Fig. 1, this turns out to be possible in restricted interval of $R$. For the compositions of Fig. 1 the available interval is about 200 to $500 \AA$.

\section{References}

[1] A.I. Ekimov, Al.L. Efros, Solid State Commun. 56, 921 (1985).

[2] M.A. Reed, J.N. Randall, R.J. Aggarwal, R.J. Matyi, T.M. Moore, A.E. Wetsel, Phys. Rev. Lett. 60, 535 (1988).

[3] Ch. Sikorski, U. Merkt, Phys. Rev. Lett. 62, 2164 (1989).

[4] G. Nimtz, B. Schlicht, Narrow Gap Semiconductors, Springer Tracts in Modern Physics, Vol. 98, Springer, Berlin 1983.

[5] B.A. Volkov, O.A. Pankratov, Pis'ma Zh. Eksp. Tcor. Fiz. 42, 115 (1985).

[6] L.A. Fal'kovskii, Zh. Eksp. Teor. Fiz. 68, 1529 (1975). 